\title{
Methoprene-tolerant 1 regulates gene transcription to maintain insect larval status
}

\author{
Wen-Li Zhao, Chun-Yan Liu, Wen Liu, Di Wang, Jin-Xing Wang and Xiao-Fan Zhao \\ The Key Laboratory of Plant Cell Engineering and Germplasm Innovation, Ministry of Education, \\ Shandong Provincial Key Laboratory of Animal Cells and Developmental Biology, School of Life Sciences, \\ Shandong University, Jinan 250100, China
}

Correspondence should be addressed to X-F Zhao

Email

xfzhao@sdu.edu.cn

\begin{abstract}
Insect molting and metamorphosis are regulated by two hormones: 20-hydroxyecdysone (20E) and juvenile hormone $(\mathrm{JH})$. The hormone $20 \mathrm{E}$ regulates gene transcription via the nuclear receptor EcR to promote metamorphosis, whereas $\mathrm{JH}$ regulates gene transcription via its intracellular receptor methoprene-tolerant (Met) to prevent larval-pupal transition. However, the function and mechanism of Met in various insect developments are not well understood. We propose that Met1 plays a key role in maintaining larval status not only by promoting $\mathrm{JH}$-responsive gene transcription but also by repressing 20E-responsive gene transcription in the Lepidopteran insect Helicoverpa armigera. Met1 protein is increased during feeding stage and decreased during molting and metamorphic stages. Met 1 is upregulated by $\mathrm{JH}$ III and a low concentration of $20 \mathrm{E}$ independently, but is downregulated by a high concentration of $20 \mathrm{E}$. Knockdown of Met1 in larvae causes precocious pupation, decrease in $\mathrm{JH}$ pathway gene expression, and increase in 20E pathway gene expression. Met1 interacts with heat shock protein 90 and binds to $\mathrm{JH}$ response element to regulate Krüppel homolog 1 transcription in $\mathrm{JH}$ III induction. Met1 interacts with ultraspiracle protein 1 (USP1) to repress 20E transcription complex ECRB1/USP1 formation and binding to ecdysone response element. These data indicate that $\mathrm{JH}$ via Met1 regulates $\mathrm{JH}$ pathway gene expression and represses $20 \mathrm{E}$ pathway gene expression to maintain the larval status.
\end{abstract}
Key Words
- methoprene-tolerant 1
- juvenile hormone
- gene transcription
- 20-hydroxyecdysone

Journal of Molecular
Endocrinology
(2014) 53, 93-104

\section{Introduction}

Insect growth, molting, and metamorphosis are coordinately regulated by two hormones, namely juvenile hormone (JH) and 20-hydroxyecdysone (20E): 20E promotes molting and metamorphosis and $\mathrm{JH}$ prevents larval-adult transition until insects have attained an appropriate stage (Riddiford et al. 2003). The hormone 20E binds to its nuclear receptor EcR and forms a transcription complex with the heterodimeric partner ultraspiracle protein (USP) in Drosophila (Yao et al. 1992). The EcR/USP transcription complex binds to ecdysone response element (EcRE) to promote gene transcription in the 20E pathway in Drosophila (Riddihough \& Pelham 1987), such as transcription factor Broad (Br) that initiates metamorphosis in Manduca sexta (Zhou \& Riddiford 2001) and transcription factor hormone receptor 3 (HR3) to promote molting and metamorphosis in $M$. sexta (Lan et al. 1999).

Methoprene-tolerant (Met) gene is cloned in a Drosophila melanogaster mutant that showed resistance to toxic doses of JH or JH analog (Ashok et al. 1998). Met protein

Published by Bioscientifica Ltd 
has a typical basic helix-loop-helix Per-ARNT-Sim (bHLH-PAS) domain. As Met binds to JH III with a high affinity through its C-terminal PAS domain (Charles et al. 2011) and regulates gene transcription in the JH pathway in Tribolium, Met is considered as the JH intranuclear receptor (Jindra et al. 2013). In a recent paper, De Loof et al. have advanced arguments in favor of the view that the still missing membrane receptor of $\mathrm{JH}$ is probably nothing else than the $\mathrm{Ca}^{2+}$-homeostasis system of cells. Indeed, at least some isoforms of $\mathrm{Ca}^{2+}$-ATPase have a binding site for farnesol-like sesquiterpenoids, the family of compounds to which JH belongs. These authors think that a low cytoplasmic $\mathrm{Ca}^{2+}$ concentration is causal to the status quo effect of JH (De Loof et al. 2014).

Met forms homodimers with Met or germ cell expressed (Gce) without hormone regulation in Drosophila (Godlewski et al. 2006). JH regulates the formation of the complex of Met with the steroid co-activator p160/SRC in Tribolium castaneum (Zhang et al. 2011, Jindra et al. 2013), which is known as FISC in Aedes aegypti (Li et al. 2011), Taiman in D. melanogaster (Charles et al. 2011), and Ncoa/Src1/p160 in mammals (Beischlag et al. 2002). In A. aegypti, Met interacts with Cycle (CYC) (Shin et al. 2012) and BFtz-F1 (Zhu et al. 2003) by the induction of JH. Met2 interacts with SRC and binds to JH response element (kJHRE) in the Krüppel homolog 1 gene (Krh1) to promote Krh1 transcription to repress metamorphosis in Bombyx mori (Kayukawa et al. 2012). However, Met is also involved in the 20E pathway. Met RNAi prevents tissue remodeling, which results in lethality and disruption of the 20E-triggered transcriptional cascade reaction in B. mori (Guo et al. 2012). In Drosophila, the overexpression of Met in fat body results in precocious and enhanced programmed cell death and cell dissociation (Liu et al. 2009). Met can also interact with EcR and USP in insect cells in an ecdysteroid or in a JH-regulation-independent manner, suggesting that the interaction among Met, EcR, and USP mediates the crosstalk between these two important hormones (Bitra $\&$ Palli 2009). Therefore, the basic function of Met in various insects is not really clear.

Helicoverpa armigera (Noctuidae, Lepidoptera) is a severe agricultural pest throughout the world. We reveal that Met1 has an important function in maintaining the larval status in this insect. Knockdown of Met1 causes precocious pupation by repressing the $\mathrm{JH}$ pathway gene expression and promoting 20E pathway gene expression. The mechanism used by Met1 in controlling 20E and JH pathways is studied in this work.

\section{Materials and methods}

\section{Experimental animal}

H. armigera larvae were raised on the artificial diet, which was made from powder of wheat germs and soybeans at $27^{\circ} \mathrm{C}$ with $60-70 \%$ relative humidity. The illumination condition consisted of $14 \mathrm{~h}$ light:10 h darkness cycles in an insectarium (Zhao et al. 1998).

\section{$\boldsymbol{H}$. armigera epidermal cell line culture}

The $H$. armigera epidermal cell line (HaEpi) was established in our laboratory (Shao et al. 2008). Cells were cultured as a loosely attached monolayer and maintained at $27^{\circ} \mathrm{C}$ in tissue culture flasks. The tissue culture flasks had an area of $25 \mathrm{~cm}^{2}$ with $4 \mathrm{ml}$ of antibiotic-free Grace's medium supplemented with 10\% heat-inactivated fetal bovine serum (FBS). Cells were subcultured weekly to a near confluent monolayer.

\section{Bioinformatic analyses of Met1}

The translation and prediction of Met1 were analyzed by ExPASy Proteomics Server (http://www.expasy.ch/tools/). The nucleotide sequence of Met1 has been submitted to GenBank with GenBank Number: KJ184572. Protein domain prediction was performed using SMART (http:// smart.embl-heidelberg.de/). The phylogenetic tree was obtained using the neighbor-joining method in MEGA 3.1 (http://www.megasoftware.net/).

\section{Recombinant expression and polyclonal antibody preparation}

The fragment of Met1 81 aa-277 aa was expressed in Escherichia coli Rosetta host cells by the vector pGEX-4T-1, as inclusion body with the primers (sequence-Met1-F1BamHI: TACTCAGGATCCCGGAGCCAATACAACTCT; Met1-R1-XhoI: TACTCACTCGAGTCAATTCCCACTGGATGA). Protein was purified by SDS-PAGE. The antiserum was prepared by the method described previously (Dong et al. 2013). The specificity of the antiserum was determined by western blot analysis.

\section{Quantitative real-time RT-PCR}

Total RNA of larvae was extracted using Unizol reagent (Biostar, Shanghai, China). The RNA was reverse transcribed into the first-strand cDNA as the template for quantitative real-time RT-PCR (qRT-PCR) with the primers

Published by Bioscientifica Ltd. 
listed in Supplementary Table S1, see section on supplementary data given at the end of this article. The $10 \mu \mathrm{l}$ mixture, which was used to perform qRT-PCR, comprised $5 \mu \mathrm{l}$ SsoFast EvaGreen Supermix (Bio-Rad), $1 \mu \mathrm{l}$ cDNA (1:50 diluted), $2 \mu \mathrm{l}$ of $1 \mu \mathrm{mol} / \mathrm{l}$ forward primer, and $2 \mu \mathrm{l}$ of $1 \mu \mathrm{mol} / 1$ reverse primer in $1 \mathrm{ml}$ FBS-free Grace's medium for $12 \mathrm{~h}$. $\beta$-actin was used for quality control. Data were analyzed using the following formula: $R=2^{-(\Delta C t \text { sample }-\Delta C t \text { control })}$, where $R$ is the relative expression level, $\Delta C t$ sample is the difference between the $C t$ of the gene and the average $\beta$-actin in the experimental sample, and $\Delta C t$ control the difference between the $C t$ of the gene and the average of $\beta$-actin in the control sample.

\section{Hormone treatment on larvae}

Stock solutions $(20 \mathrm{mM})$ of $20 \mathrm{E}$ and JH III (Sigma) were prepared in dimethyl sulfoxide (DMSO). The 20E and JH III stock solute were diluted to $0.1 \mathrm{mg} / \mathrm{ml}$ with PBS (10 mM $\mathrm{Na}_{2} \mathrm{HPO}_{4}, 1.8 \mathrm{mM} \mathrm{KH} \mathrm{PO}_{4}, 140 \mathrm{mM} \mathrm{NaCl}$, and $2.7 \mathrm{mM}$ $\mathrm{KCl}, \mathrm{pH} 7.4)$. The hormone $20 \mathrm{E}$ or JH III $(0.1 \mathrm{mg} / \mathrm{ml})$ was injected in the 6 th instar $6 \mathrm{~h}$ larvae $(500 \mathrm{ng} / \mathrm{larva})$. The untreated larvae were injected with an equivalent amount of DMSO.

\section{Western blot analysis}

Tissues were homogenized in Tris-buffered saline (TBS, $50 \mathrm{mM}$ Tris- $\mathrm{HCl}, \mathrm{pH} 7.5,150 \mathrm{mM} \mathrm{NaCl}$ ), and protein quantification was performed using a $\beta$-actin antibody. The proteins were separated in SDS-PAGE and then transferred onto a nitrocellulose membrane. After being blocked with $2 \%$ non-fat milk in TBS for $1 \mathrm{~h}$ at room temperature, the membrane was incubated with the antibody for $12 \mathrm{~h}$ at $4{ }^{\circ} \mathrm{C}$. The membrane was washed with TBST $(0.1 \%$ Tween 20 in TBS) for 15 min thrice. Subsequently, the blot was probed with the HRP-conjugated goat anti-rabbit IgG (1:10 000 in the blocking solution). To visualize the peroxidase activity, 4-chloro-1-naphthol was used as a HRP substrate.

\section{RNAi in the HaEpi cell line and larvae}

MEGA-script RNAi Kit (Ambion, Inc., Austin, TX, USA) was used to synthesize dsRNA with the T7 promoter-containing PCR primers (Met1-RNAi-F and Met1-RNAi-R sequence in Supplementary Table S1). HaEpi was cultured to $80 \%$ confluence at $27^{\circ} \mathrm{C}$ in Grace's medium supplemented with $10 \%$ FBS. Approximately $1 \mu \mathrm{g}$ dsRNA and $125 \mu$ FBSfree Grace's medium with $8 \mu \mathrm{l}$ lipofectamine were mixed to incubate cells in $1 \mathrm{ml}$ FBS-free Grace's medium for $12 \mathrm{~h}$.
Subsequently, cells were cultured in Grace's medium supplemented with FBS for $12 \mathrm{~h}$ and incubated with 20E or JH III for $12 \mathrm{~h}$. For the RNAi experiment in larvae, dsRNA was diluted in nuclease-free water to $100 \mathrm{ng} / \mu \mathrm{l}$. Approximately $5 \mu \mathrm{l}$ of dsRNA were injected in a sixth instar $6 \mathrm{~h}$ larva. The control larvae were injected with $5 \mu \mathrm{l} d s G F P$ individually. Experiments were carried out in 30 larvae and repeated thrice for statistical analysis.

\section{Hematoxylin-eosin staining}

The dewaxed histological sections were stained with hematoxylin for $10 \mathrm{~min}$ at room temperature. The sections were washed consecutively with running water and Scott liquid for $1 \mathrm{~min}$. The sections were then differentiated with $1 \%$ hydrochloric acid in alcohol for $20 \mathrm{~s}$, washed with Scott liquid for $1 \mathrm{~min}$, stained with $0.5 \%$ water-soluble eosin dye solution for $30 \mathrm{~s}$, and washed with running water. After sealing, the sections were observed under an Olympus BX51 fluorescence microscope.

\section{Co-immunoprecipitation}

The open reading frame (ORF) of the genes was cloned into the plEX-4-vector fused with red fluorescent protein (RFP) or green fluorescent protein (GFP) using the primers (Supplementary Table S1) respectively. USP1-RFP-His, EcRB1-His, Met1-GFP-His, and Met1-RFP were overexpressed in HaEpi cells via transfection with Cellfectin II Reagent (Invitrogen) according to the method described previously (Hou et al. 2012). After $48 \mathrm{~h}$ of culture, cells were incubated with $1 \mu \mathrm{M} 20 \mathrm{E}$ or JH III for $2 \mathrm{~h}$. DMSO treatment was used as the control, and RFP-His was expressed as the control. The proteins were extracted from cells using the RIPA buffer, three wells of six well plate/ $800 \mu \mathrm{l}(0.1 \mathrm{M}$ Tris- $\mathrm{HCl}$ buffer, pH 8.0 containing $0.15 \mathrm{M}$ $\mathrm{NaCl}$, and $1 \% \mathrm{NP}-40$ ) and harvested by centrifugation at $12000 \mathrm{~g}$ for $20 \mathrm{~min}$ at $4{ }^{\circ} \mathrm{C}$. Approximately $30 \mu \mathrm{l}$ to $40 \mu \mathrm{l}$ of supernatant were used as input proteins. The rest of the supernatant was added the antiserum against Met1 (1:100), incubated for $3 \mathrm{~h}$ with gentle shaking at $4{ }^{\circ} \mathrm{C}$, and then incubated with $40 \mu$ l Protein A resin for $3 \mathrm{~h}$ with gentle shaking at $4{ }^{\circ} \mathrm{C}$. After centrifugation at $12000 \mathrm{~g}$ for $10 \mathrm{~min}$ at $4{ }^{\circ} \mathrm{C}$, the supernatant was discarded. After being washed with PBS, the resin was treated with the SDS-PAGE loading buffer and boiled for $10 \mathrm{~min}$ for western blot analysis with various antibodies. The molecular masses of the proteins are shown in Supplementary Figure S3 (see section on supplementary data given at the end of this article).

Published by Bioscientifica Ltd. 


\section{Electrophoretic mobility shift assay}

Various proteins were overexpressed in HaEpi cells. The nuclear proteins were extracted from HaEpi cells and diluted to $1 \mu \mathrm{g} / \mu \mathrm{l}$. T. castaneum Krh1-JHRE-digoxin-labeled doublestranded probe contains E-box (sequences: 5'-GCGGTGGGCCTCCACGTGTCGAACG-3' and 3'-CGTTCGACACGTGGAGGCCCACCGC-5'). The E-box 'CACGTG' is conserved in Apis mellifera, Acyrthosiphon pisum, B. mori, D. melanogaster, N. vitripennis, and T. castaneum (Kayukawa et al. 2012). H. armigera HR3-EcRE (Liu et al. 2014) digoxinlabeled double-stranded probe (sense $5^{\prime}$-ggtcccggggtcaatgaactgctgtga- $3^{\prime}$ and antisense $5^{\prime}$-tcacagcagttcattgacccoggacc- $3^{\prime}$ ) was synthesized by Shengong (Shanghai, China). The probes were incubated with nuclear proteins for $20 \mathrm{~min}$ at room temperature in a binding buffer (Beyotime, Shanghai, China). The detailed method was described in our previous paper (Liu et al. 2014). The $100 \mathrm{fmol}$ probes were incubated with $5 \mu \mathrm{g}$ nuclear proteins for $20 \mathrm{~min}$ at room temperature in $15 \mu \mathrm{l}$ binding buffer (Beyotime, Shanghai, China). A fifty-fold amount of non-labeled-probe was used as specific competitor. For antibody mediated super shift assay, antiserum (1:2000) was incubated with the nuclear proteins in reaction mixture for $20 \mathrm{~min}$, followed by incubation with a digoxin-labeled probe for another $20 \mathrm{~min}$. The proteinDNA complex was separated from the free probe in $5.75 \%$ non-denaturing polyacrylamide gel.

\section{Results}

The full cDNA sequence of Met1 is $2410 \mathrm{bp}$, with an ORF of $1581 \mathrm{bp}$, and encodes 526 amino acid proteins with a molecular mass of $59 \mathrm{kDa}$. Met 1 has the typical characteristics of b-HLH-PAS family proteins with four structural domains of b-HLH, two PAS, and one PAC. Met1 of

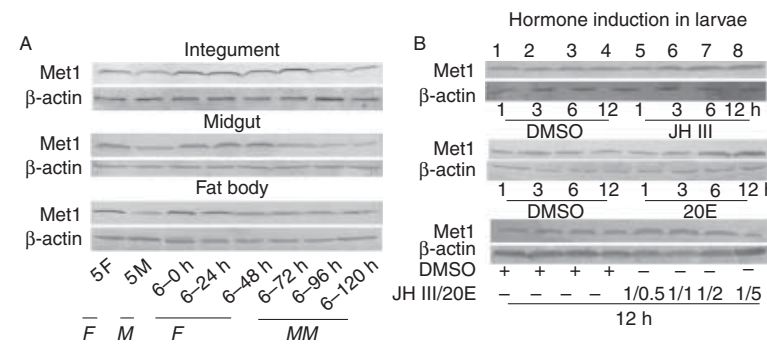

\section{Figure 1}

Western blot analysis showing Met1 expression profiles during larval development. The specificity of the antibody against $H$. armigera Met1 is shown in Supplementary Figure S2, see section on supplementary data given at the end of this article. (A) Profile of Met1 expression in the integument, midgut, and fat body. $F$, feeding; $M$, molting; $M M$, metamorphic molting. $\beta$-actin was used as the loading control. (a) Quantification of Met1 expression in (A) using the ImageJ software
H. armigera is similar to Met1 of B. mori (Supplementary Figure S1, see section on supplementary data given at the end of this article). HLH is a DNA-binding domain (Murre et al. 1989). PAS is used as a signal sensor domain (Taylor \& Zhulin 1999). PAC is a motif C-terminal to PAS, which is proposed to contribute to the PAS domain fold by SMART analysis (http://smart.embl-heidelberg.de/).

\section{Met1 expression level increased during feeding but decreased during metamorphosis}

To study the function of Met1, we examined the expression profile during development from fifth instar to prepupae. Met1 showed a higher expression level at the fifth instar feeding stage $(\mathrm{F})$ and the sixth instar feeding stage $(\mathrm{F})$ compared with the fifth instar molting stage (M) and the metamorphic molting stage (MM) in the integument, midgut, and fat body (Fig. 1A and a). This expression pattern suggests that Met1 expression level is regulated during larval development.

Considering that $\mathrm{JH}$ titer is higher during feeding and $20 \mathrm{E}$ is higher during molting and metamorphosis in $M$. sexta (Riddiford et al. 2003), we injected sixth instar $6 \mathrm{~h}$ larvae with JH III and 20E to analyze the hormone regulation in Met1 expression. Results showed that 20E and JH III upregulated the expression of Met 1 in the midgut independently. However, the higher concentration of $20 \mathrm{E}$ repressed Met1 expression (Fig. 1B and b). These results suggest that Met1 expression is coordinately regulated by $\mathrm{JH}$ and $20 \mathrm{E}$.

\section{Knockdown of Met1 causes precocious pupation by changing the gene expression}

Met1 was knocked down by injecting dsRNA of Met1 into 6 th instar $6 \mathrm{~h}$ larvae to examine the function of Met1 in

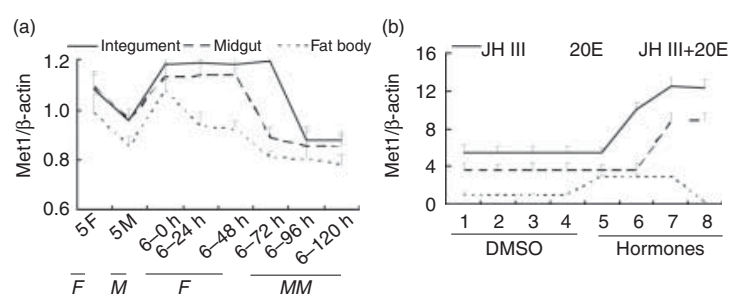

(National Institutes of Health, http://imagej.nih.gov/ij/download.html) based on three independent experiments. (B) The 6 th instar $6 \mathrm{~h}$ larvae were injected with JH III, $20 \mathrm{E}$ (500 ng/larva), or JH III (500 ng/larva) plus 20E (250, 500,1000 , and $2500 \mathrm{ng} / \mathrm{larva}$ ) for $1,3,6$, and $12 \mathrm{~h}$, respectively, and the midgut proteins were examined. An equal volume of DMSO is the hormone solvent control. $\beta$-actin was used as the loading control. (b) Quantification analysis of hormone induction of Met1 in B using the ImageJ software.

Published by Bioscientifica Ltd. 
the larval development. The knockdown of Met1 caused precocious pupation (Fig. 2A and B). The pupation time from 6th instar $0 \mathrm{~h}$ to pupa in dsMet1-injected larvae was $92 \mathrm{~h}$, which was $28 \mathrm{~h}$ more precocious than the $120 \mathrm{~h}$
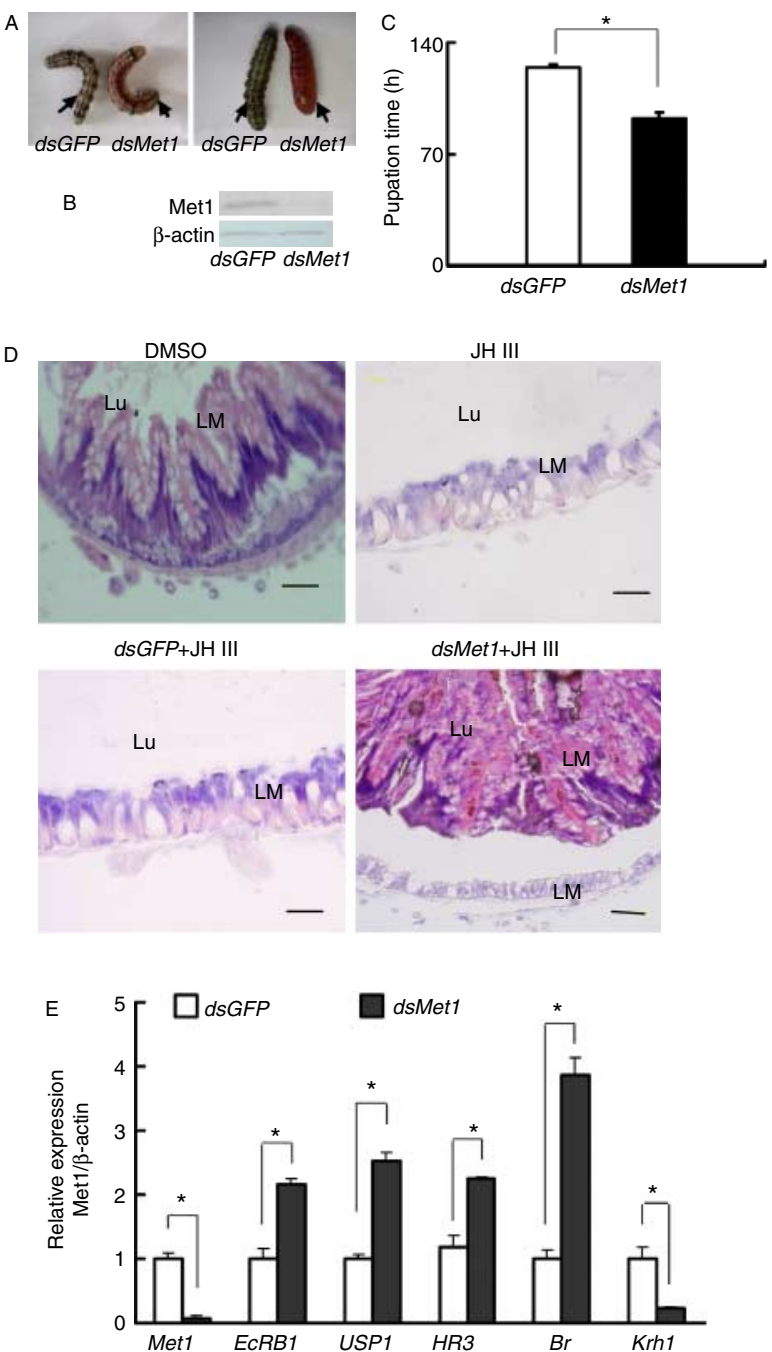

Figure 2

Knockdown of Met1 causes precocious pupation by altering the gene expression. (A) Phenotypes after knockdown of Met1 (injection of dsMet1 to 6 th instar $6 \mathrm{~h}$ larvae, $500 \mathrm{ng} / \mathrm{larva}$, twice in $48 \mathrm{~h}$ interval). (B) Western blot analysis showed the efficacy of Met1 knockdown. (C) Statistical analysis of the pupation time of $50 \%$ larvae $\left(P_{50}\right)$ after Met1 knockdown (30 larvae, three triplicates) by Student's $t$-test. (D) HE staining showed the midgut remodeling $70 \mathrm{~h}$ after first $d s M e t 1$ injection. DMSO as the JH III solvent control; $\mathrm{JH}$ III injection; dsGFP and JH III injection (12 h after dsGFP injection); and dsMet1 and JH III injection (12 h after dsMet1 injection). A total of $500 \mathrm{ng}$ of dsRNA or JH III per larva. Lu, midgut lumen; LM, larval midgut; IM, imaginal midgut. Bar is $50 \mu \mathrm{m}$. (E) Gene expression in the midgut after Met1 knockdown in larvae (500 ng dsMet1/larva, extracted RNA $48 \mathrm{~h}$ after first injection). Asterisks indicate significant differences between the groups $(P<0.05)$ by Student's $t$-test based on three independent experiments. A full colour version of this figure is available at http://dx.doi.org/10.1530/JME-14-0019. in $d s G F P$-injected control larvae (Fig. 2C). These results suggest that Met1 is involved in maintaining the larval status.

$\mathrm{JH}$ III was injected in the 6th instar $6 \mathrm{~h}$ larval hemocoel to examine the involvement of Met1 in $\mathrm{JH}$ III function of preventing metamorphosis. In the DMSO-injected control, the midgut performed normal remodeling by separating from the imaginal midgut $70 \mathrm{~h}$ after the first injection. By contrast, injection of JH III prevented midgut remodeling. $d s G F P$ injection did not repress the JH III-prevented midgut remodeling. However, dsMet1 injection repressed the JH III-prevented midgut remodeling (Fig. 2D). These results suggest that Met1 is involved in the JH III function to prevent metamorphosis.

To address the mechanism of Met1 knockdown causing precocious metamorphosis, we examined a set of gene expression after Met1 knockdown in larvae, including the 20E pathway genes EcRB1, USP1, HR3, and $B r$, and $\mathrm{JH}$ pathway gene Krh1 using qRT-PCR. EcRB1, USP1, HR3, and $\mathrm{Br}$ significantly increased in the midgut after Met1 knockdown compared with the $d s G F P$-injected control. However, Krh1 was downregulated after Met1 knockdown (Fig. 2E). These results suggest that Met 1 knockdown caused precocious metamorphosis because of the change in gene expression, and Met1 is a promoter of $\mathrm{JH}$ pathway gene expression and a repressor of $20 \mathrm{E}$ pathway gene expression.

\section{Knockdown of Met1 in HaEpi cells represses gene expression in JH pathway and promotes gene expression in $20 \mathrm{E}$ pathway}

To confirm the conclusion regarding the Met1 knockdown in larvae, the function of Met1 in JH and 20E pathways was examined in HaEpi cells by RNAi. JH III increased expression of Met1, USP1, Br, and Krh1 transcripts. However, after knockdown of Met1, JH III could not increase expression of these gene transcripts (Fig. 3A). By contrast, 20E increased expression of Met1, EcRB1, USP1, HR3, Br, and Krh1 transcripts in cells. However, after knockdown of Met1, 20E induced expression of EcRB1, USP1, HR3, and $B r$ to a greater extent (Fig. 3B). These results confirm that JH via Met1 promotes USP1, $B r$, and $K r h 1$ expression, and Met1 inhibits 20E-induced EcRB1, USP1, HR3, and Br expression.

\section{Overexpression of Met1 in HaEpi cells promotes JH III-induced gene expression and represses 20E-induced gene expression}

To corroborate the data in Met1 knockdown in HaEpi cells, we induced overexpression of Met1 in HaEpi cells by fusing

Published by Bioscientifica Ltd 

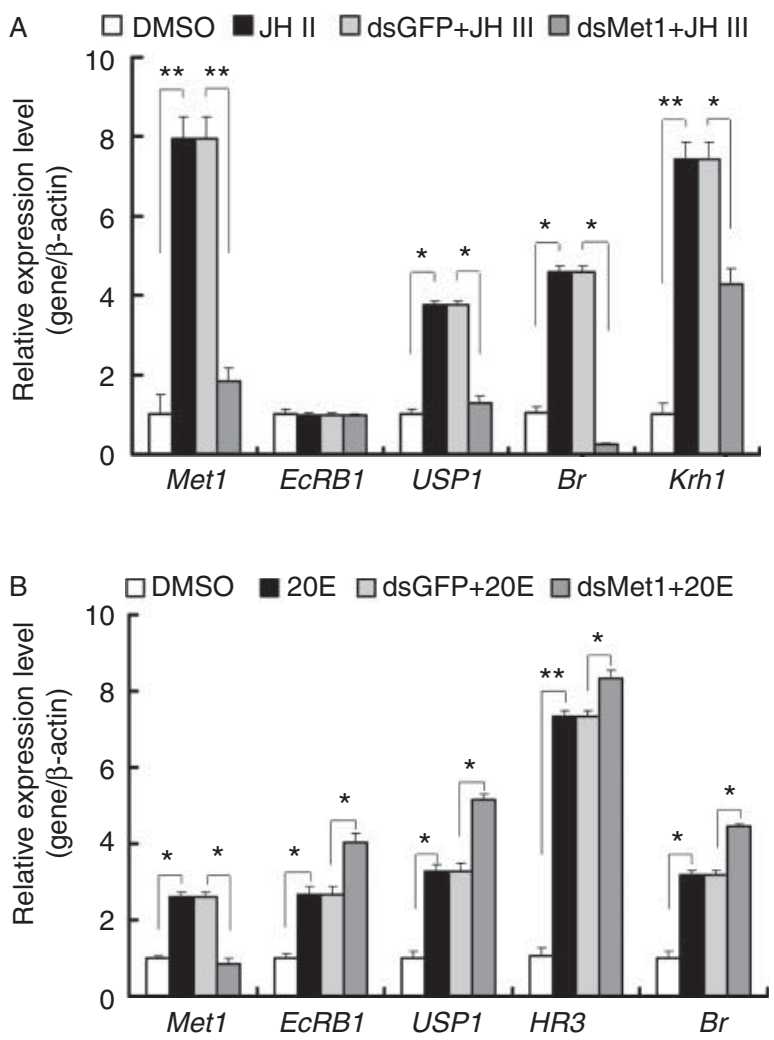

\section{Figure 3}

qRT-PCR analyses showing gene expression in HaEpi cells after Met1 knockdown. (A) Decreased gene expression in JH induction. (B) Increased gene expression in $20 \mathrm{E}$ induction $(1 \mu \mathrm{g} / \mathrm{ml}$ of dsMet, $1 \mu \mathrm{M} \mathrm{JH}$ or $20 \mathrm{E}$ for $12 \mathrm{~h}$ ). Asterisks indicate the significant differences between the groups $(P<0.05)$ by Student's $t$-test based on three independent experiments.

it at the front of RFP in the pIEx-RFP-His vector. The overexpression of RFP-His alone is used as the nonspecific protein control. The red fluorescent signal was detected in the entire cell in the RFP-expressing cells. However, the red fluorescent signal was detected in the nucleus in Met1-RFPexpressing cells (Fig. 4A). The expression of Met1-RFP-His was confirmed by western blot analysis (Fig. 4B). The overexpressed Met1-RFP-His increased the JH III-induced expression levels of $U S P 1, B r$, and $K r h 1$ compared with the RFP-His control (Fig. 4C). By contrast, the overexpressed Met1-RFP-His repressed 20E-induced EcRB1, USP1, HR3, and $\mathrm{Br}$ expression (Fig. 4D). This result confirms that Met1 promotes the $\mathrm{JH}$ pathway gene expression and represses 20 E pathway gene expression.

\section{Met1 interacts with Hsp90 and binds to Krh1-JHRE}

Met binds to the E-box 'CACGTG' in the core region of kJHRE (GGCCTCCACGTG) and the E-box is conserved in six species (A. mellifera, A. pisum, B. mori, D. melanogaster,
N. vitripennis, and T. castaneum) (Kayukawa et al. 2012). To confirm that JH III regulates Krh1 transcription via Met, we overexpressed Met1-RFP in HaEpi cells and performed an electrophoretic mobility shift assay (EMSA) experiment using kJHRE (GGCCTCCACGTG) in the Krh1 promoter of Tribolium as a probe. In the RFP-expressing cells, the nuclear extracts from normal cells, or DMSO- or 20Etreated cells could not change the mobility of kJHRE (Fig. 5A and lane 1, 2, and 3). However, the nuclear extracts from JH III-treated RFP-expressing cells changed the mobility of kJHRE (Fig. 5A and lane 4), suggesting that $\mathrm{JH}$ III-induced nuclear proteins can bind to kJHRE. In the Met1-RFP-expressing cells, the nuclear extracts from DMSO- or 20E-treated cells could not change the mobility of kJHRE (Fig. 5A and lanes 5 and 6). However, the nuclear extracts from JH III-treated cells changed the mobility of kJHRE and increased the amount of mobilized kJHRE compared with RFP-expressing control cells (Fig. 5A and lane 7 compared with lane 4). When the 50-fold excess of unlabeled kJHRE was added, the amount of the mobilized kJHRE decreased (Fig. 5A and lane 8 compared with lane 7), suggesting that Met1-RFP binds to kJHRE. After the addition of the antibodies against either $H s p 90$ or Met1, the mobilized kJHRE band disappeared (Fig. 5A and lane 9 and 10). The data were statistically analyzed (Fig. 5B). These results confirm that Met1-RFP binds to kJHRE, and Hsp90 is necessary for this binding.

Given that Met1 can interact with Hsp90 to regulate the gene expression in the JH pathway in $\mathrm{H}$. armigera (Liu et al. 2013), Co-IP was conducted to confirm the interaction between Met1 and Hsp90 in JH induction. Hsp90 was co-precipitated by an anti-Met1 antibody from the JH III-treated cells, but not from the 20E-induced cells (Fig. 5C and D). These findings suggest that Met 1 interacts with Hsp90 and binds to kJHRE to promote Krh1 transcription in the JH pathway.

\section{Met1 represses EcRB1-USP1 interaction}

Considering that the overexpression of Met1 repressed 20E-induced gene transcription, we performed an EMSA experiment by examining the binding of EcRB1 to EcRE in $H$. armigera HR3 (5'-GGGGTCAATGAACTG-3' and $3^{\prime}$-CAGTTCATTGACCCC-5') (Liu et al. 2014) to investigate the mechanism. In the RFP-expressing control cells, when the nuclear extracts from DMSO- or JH III-induced RFP-expressing cells were incubated with Dig-EcRE, no mobilized Dig-EcRE was detected (Fig. 6A and lanes 2 and 4). However, Dig-labeled EcRE was mobilized by the nuclear extracts from 20E-treated cells (Fig. 6A and lane 3

Published by Bioscientifica Ltd. 

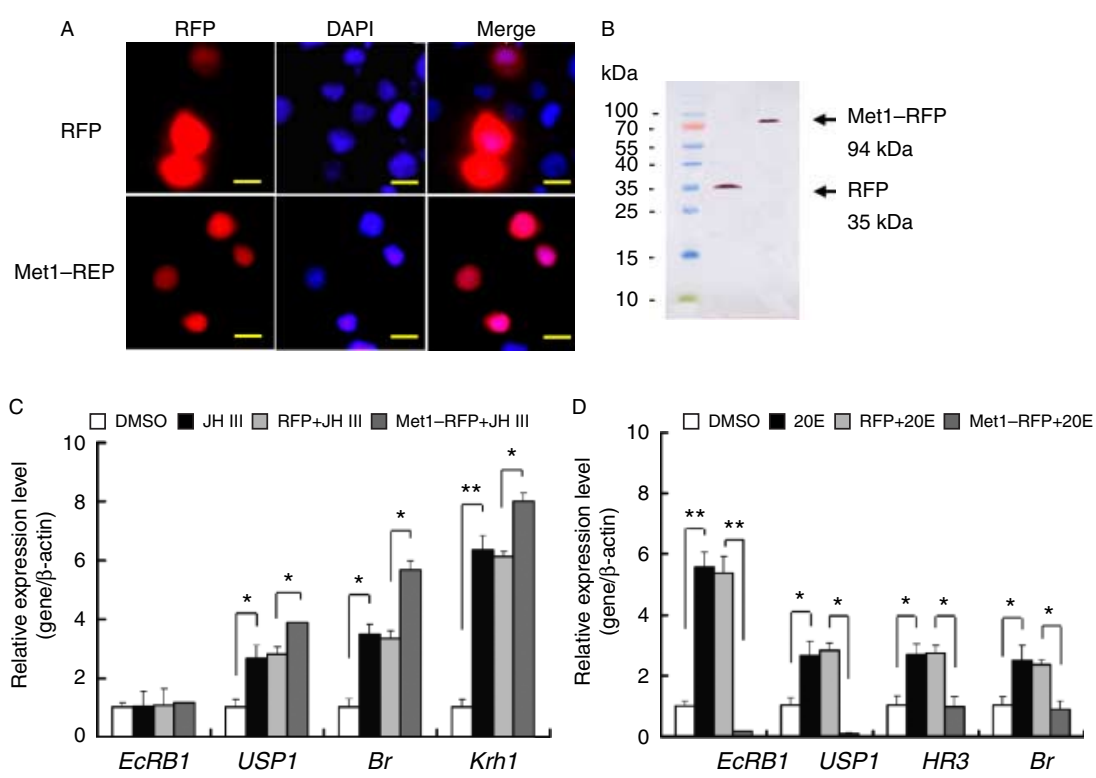

\section{Figure 4}

The overexpression of Met1 in HaEpi cells promotes $\mathrm{JH}$-induced gene expression and represses $20 \mathrm{E}$-induced gene expression. (A) Overexpression of Met1-RFP-His in HaEpi cells. RFP (red); Met1-RFP (red); DAPI indicates the cell nucleus (blue); and merge is the overlapped red and blue. Bar: $20 \mu \mathrm{m}$. (B) Western blot analysis showing the expression of RFP (35 kDa) and Met1-RFP-His (94 kDa) in HaEpi cells, as detected using the

compared with lane 2), and the non-Dig-labeled EcRE competitor could compete to decrease the amount of mobilized Dig-EcRE (Fig. 6A and lane 5 compared with lane 3), suggesting 20E-induced nuclear extracts binding to the Dig-EcRE probe. The overexpressed Met1-RFP decreased the 20E-induced amount of mobilized DigEcRE (Fig. 6A and lane 7 compared with lane 3). The nonDig-labeled EcRE competitor decreased the amount of mobilized Dig-EcRE (Fig. 6A and lane 8 compared with lane 7). The antibody against Met1 increased the amount of mobilized Dig-EcRE (Fig. 6A and lane 9 compared with lane 7). JH III repressed the binding of nuclear extracts to the Dig-EcRE probe (Fig. 6A and lane 10). The EcRB1-His was overexpressed in cells to confirm 20E-induced binding of EcRB1 to EcRE. The amount of mobilized Dig-EcRE by 20E-induced nuclear extracts (Fig. 6C and lane 3) was decreased using a anti-His antibody that recognizes EcRB1-His (Fig. 6C and lane 4), indicating that the nuclear protein in the nuclear extracts binding to EcRE was EcRB1. Statistical analysis showed the significant difference in the amount of the mobilized Dig-EcRE in various treatments (Fig. 6B and D). These results suggest that Met1 represses the EcRB1 binding to EcRE.

To address the mechanism of overexpressed Met repressing the EcRB1 binding to EcRE, we induced
anti-His antibody. (C and D). JH and 20E ( $1 \mu \mathrm{M}$ for $12 \mathrm{~h})$ induction of the gene expression in Met1-RFP-His overexpressing cells by qRT-PCR analysis respectively. Asterisks indicate significant differences between the groups $(P<0.05)$ by Student's $t$-test based on three independent experiments. A full colour version of this figure is available at http://dx.doi.org/10.1530/ JME-14-0019.

overexpression of EcRB1-His, USP1-RFP, and Met1-GFP in HaEpi cells simultaneously. Three proteins were equally expressed in cells, as shown in the input proteins (Fig. 6E, Input). From the co-immunoprecipitates (Co-IP) by antiRFP that precipitated USP1-RFP as well as the interacting proteins, Met1-GFP and USP1-RFP, were detected either in the DMSO- or JH III-treated cells, whereas, EcRB1-His was detected together with Met1-GFP and USP1-RFP in 20Etreated cells. After addition of JH III to cells, the amount of 20E-induced EcRB1-His was reduced and that of Met1GFP was increased (Fig. 6E, Co-IP). Statistical analysis revealed that the differences in the protein amounts in various treatments were significant (Fig. $6 \mathrm{E}$ and F). These results suggest that Met1 interacts with USP1-independent hormone regulation; however, 20E promotes the interaction between EcRB1 and USP1, and JH increases the interaction between USP1 and Met1.

Given that USP is phosphorylated by $20 \mathrm{E}$ induction and the phosphorylation of USP is essential for its function in gene transcription (Sun \& Song 2006, Wang et al. 2012), the phosphorylation status of USP1 was examined by a 7.5\% low-concentration SDS-PAGE gel to demonstrate the mechanism of EcRB1 and USP1 interaction in 20E induction. The overexpressed USP1-His in HaEpi cells partially increased the molecular mass $6 \mathrm{~h}$ after $20 \mathrm{E}$

Published by Bioscientifica Lto. 
A

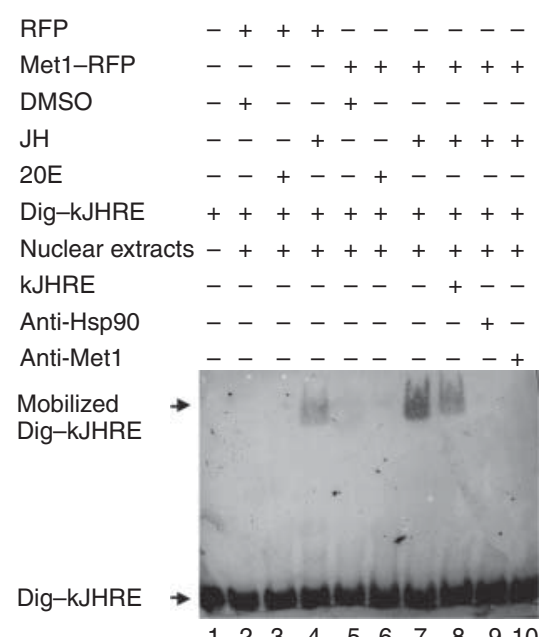

B

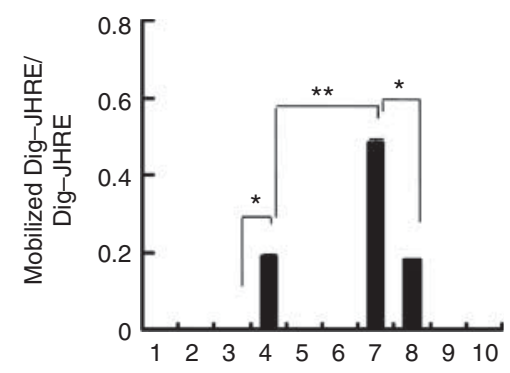

C
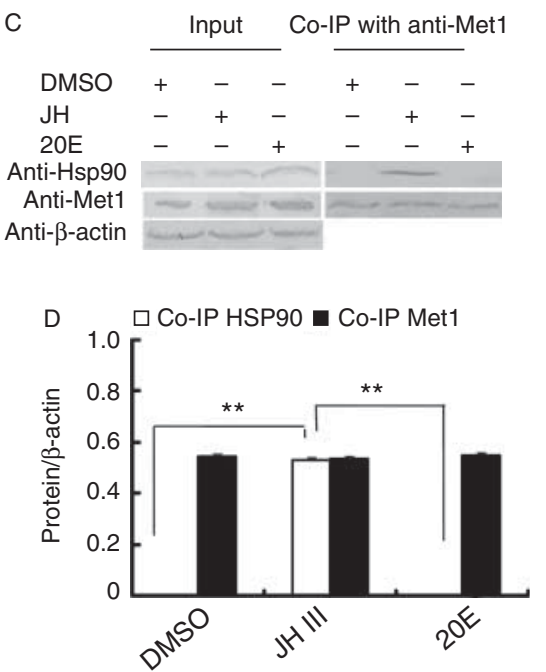

Figure 5

Met1 interacts with Hsp90 and binds to KJHRE. (A) EMSA analysis of Met1-Hsp90 binding to KJHRE. Lane 1, normal cells; lanes 2, 3, and 4, RFP-overexpressing cells treated with DMSO, 20E, or JH III ( $1 \mu \mathrm{M}$ for $6 \mathrm{~h})$; lanes 5, 6, 7, 8, 9, and 10, Met1-RFP-overexpressing cells treated with DMSO, 20E, JH III (1 $\mu \mathrm{M}$ for $6 \mathrm{~h}$ ), competitor kJHRE, or antibodies respectively. KJHRE, as a non-labeled competitor, is in 50-fold excess of the labeled probe; and anti-Met1 and anti-Hsp90 are the rabbit polyclonal antibodies against $H$. armigera. (B) Statistical analysis of the data in (A) Asterisks indicate significant differences between the groups $(P<0.05)$

treatment, compared with DMSO and JH III treatments. The increased molecular mass of USP1-His was decreased upon lambda protein phosphatase treatment, indicating that it was the phosphorylated form of USP1 (Fig. 6G). These results suggest that 20E regulates USP1 phosphorylation to interact with EcRB1, and JH III maintains USP1 nonphosphorylation to interact with Met1.

\section{Discussion}

Met is undoubtedly an important transcription factor with a crucial function in insect development. However, the function and mechanism of Met in regulating insect development are not well understood because of its complicated roles in either the $\mathrm{JH}$ pathway or the $20 \mathrm{E}$ pathway. In this research, we demonstrated that Met1 promotes JH-regulated $K r h 1$ transcription and represses 20E-induced $E c R B 1, U S P 1, B r$, and $H R 3$ transcription to maintain the larval status. The expression level of Met1 is upregulated in larval life when JH is present, i.e. during the feeding stages. It is downregulated during molting and metamorphosis, thus in stages when the $\mathrm{JH}$ titer is lowered or even zero. In these stages, the ecdysteroid titer is increased. JH mediates Met1 binding by Student's $t$-test based on three independent experiments. (C) Co-IP experiments show that $\mathrm{JH}$ regulates Met1 and Hsp90 interaction. Input: protein expression levels of $\mathrm{Hsp} 90$ and Met1 in various treated cells. Co-IP: Met1 was immunoprecipitated with an anti-Helicoverpa-Met1 antibody, whereas co-precipitated $\mathrm{Hsp} 90$ was detected by an anti-Helicoverpa-Hsp 90 antibody. $\beta$-actin was used as the loading control in the experiments. (D) Statistical analysis of the data in Co-IP in (C) Asterisks indicate significant differences between the groups $(P<0.05)$ by Student's $t$-test based on three independent experiments.

to kJHRE via interaction with Hsp90 to promote Krh1 transcription. Met1 represses EcRB1/USP1 binding to EcRE for $E c R B 1, U S P 1, B r$, and $H R 3$ transcription by interacting with USP1.

\section{Met1 promotes Krh1 transcription to maintain the larval status}

Tribolium Met functions in $\mathrm{JH}$ response to mediate the antimetamorphic effect of JH (Konopova \& Jindra 2007). Double mutation of Met and gce in Drosophila larvae totally blocks the Krh1 expression (Abdou et al. 2011). Krh1 is a JH early-inducible gene identified in D. melanogaster (Minakuchi et al. 2008). Knockdown of Tribolium Krh1 causes precocious metamorphosis (Minakuchi et al. 2009). In our study on $H$. armigera, Met1 knockdown causes precocious pupation and midgut remodeling. Correlated with the precocious pupation, Met1 knockdown repressed the Krh1 expression. These results are similar to the observations in Drosophila and Tribolium. Therefore, Met1 promotes the $\mathrm{JH}$ pathway in $H$. armigera. The function of Met1 at the level of the nucleus is the maintenance of the larval status.

Published by Bioscientifica Ltd. 
A
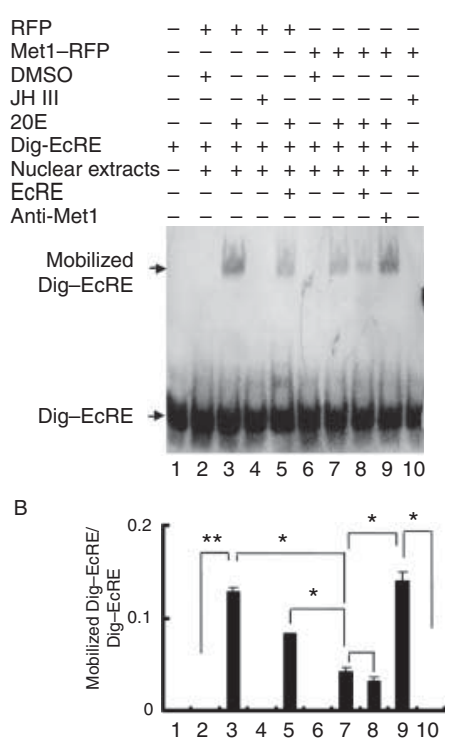

C

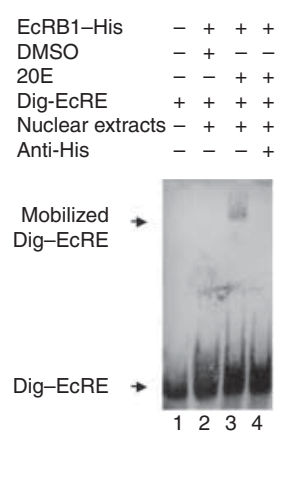

D

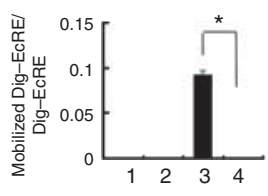

E
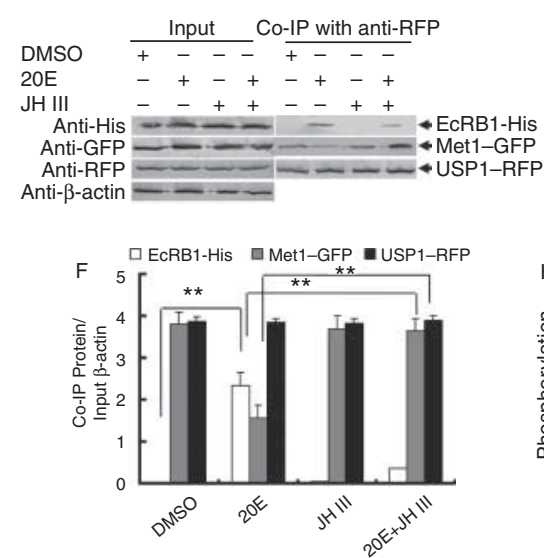

G
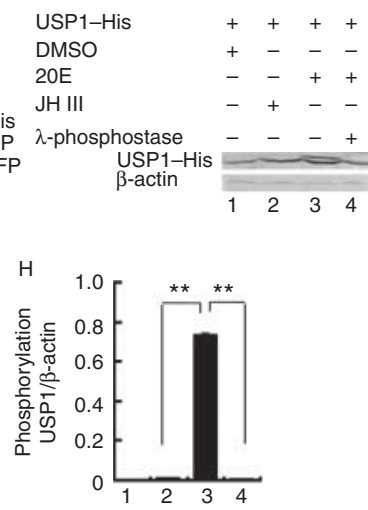

\section{Figure 6}

Overexpressed Met1 represses ECRB1 binding to ECRE. (A) EMSA analysis of nuclear protein binding to ECRE. Lane 1, the nuclear extracts from normal cells; lanes 2, 3, 4, and 5, the nuclear extracts from the RFP-overexpressing control cells treated with DMSO, 20E, JH III (1 $\mu \mathrm{M}$ for $6 \mathrm{~h})$, and a competitor (non-labeled probe ECRE, 50 -fold molar excess of Dig-labeled probe EcRE); and lanes 6, 7, 8, 9, and 10, the nuclear extracts from Met1-RFP-

overexpressing cells treated with DMSO, 20E, competitor, anti-Met1, and JH III. (B) Statistical analysis of data in (A) using Student's $t$-test (asterisks indicate significant differences $(P<0.05)$ between the groups based on three independent experiments for all the following statistical analyses). (C) EMSA analysis of ECRB1 binding to EcRE in the induction of $20 \mathrm{E}$.

(D) Statistical analysis of data in B by Student's $t$-test. (E) Co-IP showing the protein interaction among Met1, EcRB1, and USP1 (12.5\% SDS-PAGE gel). pIEX-4T1-ECRB1-His, pIEX-4T1-USP1-RFP, and pIEX-4T1-Met1-GFP

JH regulates PKC-phosphorylated Hsp90 and interaction with Met1 to participate in Krh1 expression (Liu et al. 2013). In this study, we further revealed that the complex of Hsp90 and Met1 binds to kJHRE to promote Krh1 transcription. Considering the critical function of Krh1 in the suppression of insect metamorphosis (Kayukawa et al. 2012), we proposed that JH maintains the larval status by upregulating Met 1 expression and then directing the Krh1 expression.

The fact that JH acts not only through a nuclear receptor but through a membrane receptor(s) as well should not be overlooked. According to De Loof et al. (2014), a low cytoplasmic $\mathrm{Ca}^{2+}$ concentration is causal to maintaining the larval status of holometabolous insects. At least some of the proteins that constitute the $\mathrm{Ca}^{2+}$ homeostasis system have a binding site for sesquiterpenoids, JH being one of them. At a higher $\mathrm{Ca}^{2+}$ concentration, $\mathrm{Ca}^{2+}$-induced programed cell death, a typical aspect of metamorphosis, is plasmids were co-transfected in cells. Input: anti-His detected EcRB1-His; anti-GFP detected Met1-GFP; anti-RFP detected USP1-RFP; and anti- $\beta$-actin detected $\beta$-actin in various treatments. Co-IP: USP1-RFP was immunoprecipitated with an anti-RFP monoclonal antibody. Co-precipitated ECRB1-His and Met1-GFP were detected by anti-His or anti-GFP antibodies, respectively, in cells treated with DMSO, 20E, JH III, and 20E plus JH III $(1 \mu \mathrm{M}$ for $6 \mathrm{~h})$. $\beta$-actin was used as the loading control in the experiments. (F) Statistical analysis of data in (E) by Student's $t$-test. (G) Western blot analysis showed the phosphorylation of USP1 in the induction of $20 \mathrm{E}$ (7.5\% SDS-PAGE gel). USP1-His-overexpressing cells were treated with DMSO, 20E, or JH III ( $1 \mu \mathrm{M}$ for $6 \mathrm{~h})$. The 20E-treated protein was incubated with Lambda protein phosphatase ( $\lambda$-phosphatase) with $\beta$-actin as the loading control. (H) Statistical analysis of data in (G) by Student's $t$-test.

activated. JH may play a role through the membrane receptor and protein kinase $\mathrm{C}$ in the accessory gland of male fruit flies and the follicle of vampire bugs (Sevala \& Davey 1993). However, the specific membrane receptor of $\mathrm{JH}$ needs to be elucidated.

\section{Met1 represses the transcription of a set of 20E-responsive genes to prevent metamorphosis}

Met1 represses a set of 20E-responsive gene transcription, including EcRB1, USP1, HR3, and Br, EcRB1 and USP1 form a transcription complex and bind to EcRE to initiate gene transcription (Jindra et al. 1996). HR3 is a reporter gene in the 20E pathway that is involved in molting and metamorphosis (Lam et al. 1999). Br is a metamorphosis initiator that promotes programed cell death gene expression (Cakouros et al. 2002). Knockdown of Met1 helps 20E to induce EcRB1, USP1, HR3, and $\mathrm{Br}$

Published by Bioscientifica Ltd. 


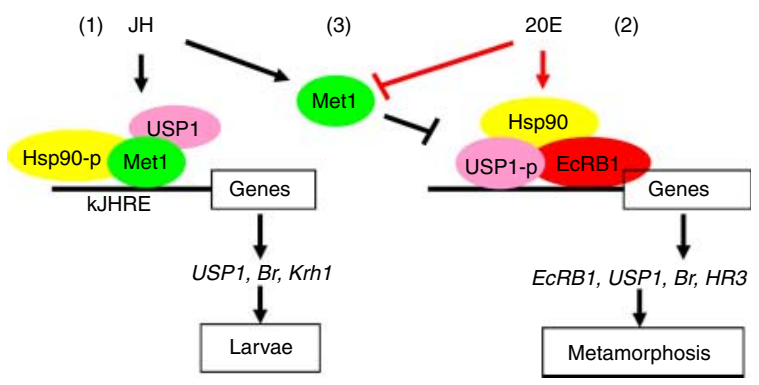

Figure 7

Chart to interpret the function and mechanism of Met1 in regulating larval development. (1) JH upregulates Met1 expression and interaction with phosphorylated Hsp90 and nonphosphorylated USP; Met1 binds to KJHRE to promote gene transcription to maintain the larval status. (2) The hormone 20E represses Met1 expression and regulates USP1 phosphorylation to interact with EcRB1; EcRB1/USP1 binds to EcRE to promote 20E-responsive gene transcription for metamorphosis. (3) Met1 interacts with USP1 to repress the interaction between ECRB1 and USP1 and its binding to ECRE. A full colour version of this figure is available at http://dx.doi.org/10.1530/ JME-14-0019.

transcription, thus promoting precocious metamorphosis, suggesting that Met1 is a repressor of the 20E pathway.

By EMSA experimental analysis, the overexpressed Met1 can repress EcRB1 binding to EcRE. This might be due to the fact that Met1 interacts with USP1 that disturbs the formation of EcRB1/USP1 complex and its binding to EcRE. USP1 is the heterodimeric partner of EcRB1 in the formation of a transcription complex for gene transcription in the $20 \mathrm{E}$ pathway. JH also binds to USP1 and participates in $\mathrm{JH}$-regulated gene transcription, suggesting its role in JH pathway (Jones \& Sharp 1997). We found that 20E regulates USP1 partially phosphorylated to interact with EcRB1. JH maintains USP1 nonphosphorylation to repress its interaction with EcRB1 and increases its interaction with Met1. However, the interaction between Met1 and USP1 may not rely on $\mathrm{JH}$ regulation because the overexpressed Met1 is co-immunoprecipitated with nonphosphorylated USP1 in DMSO-treated cells also. The 20E- or JH hormoneindependent interactions between Met and USP and between Met and EcRB1 has been reported in D. melanogaster (Bitra \& Palli 2009) and B. mori (Guo et al. 2012) respectively. Therefore, Met1 might repress 20E pathway gene transcription by repressing the formation of EcRB1/USP1 transcription complex and its binding to EcRE.

\section{Expression of Met1 is coordinately regulated by $\mathrm{JH}$ and $20 \mathrm{E}$}

In Tribolium, Met is continuously expressed throughout embryonic development (Konopova \& Jindra 2007).
In Bombyx, the developmental profiles of Met1 and Met2 from day 2 of the 4th instar to day 2 of the prepupal stage, when the 20E titer is high, have been demonstrated by Guo et al. (2012). In H. armigera, Met1 is expressed during the entire larval stage from the 5th instar stage up to the prepupal stage. However, the expression levels increased during the feeding stage and decreased at molting and later metamorphic stages. The different expression profiles in various insects may be attributed to species specificity. JH III and 20E may independently upregulate Met1 expression. However, a higher concentration of $20 \mathrm{E}$ represses Met1 expression. Thus, Met1 is coordinately regulated by the two hormones.

\section{Conclusion}

This study deepens our understanding of how interactions among a number of key transcription factors contribute, at the level of the nucleus, to the maintenance of the larval status by JH. JH upregulates the expression of Met1 and interaction with phosphorylated Hsp90 and nonphosphorylated USP1, which binds to kJHRE to promote Krh1 transcription for larval growth. The hormone 20E mediates the interaction between EcRB1 and phosphorylated USP1. Met1 interacts with USP1 to repress the formation of EcRB1/USP1 complex and its binding to EcRE and, therefore, to repress 20E-induced gene transcription and metamorphosis (Fig. 7).

The challenge for future experiments is to uncover which genes are activated by the $\mathrm{JH}$ - and 20E-dependent transcription factors. Perhaps, the transcription of genes coding for some of the proteins that make up the $\mathrm{Ca}^{2+}$ homeostasis system, which seems to play a crucial role in realizing some JH-dependent effects, is controlled this way.

\section{Supplementary data}

This is linked to the online version of the paper at http://dx.doi.org/10.1530/ JME-14-0019.

Declaration of interest

The authors declare that there is no conflict of interest that could be perceived as prejudicing the impartiality of the research reported.

\section{Funding}

This study was supported by the National Basic Research Program of China (973 Program, grant number 2012CB114101), the National Natural Science Foundation of China (grant number 31230067), and the PhD Programs Foundation of the Ministry of Education of China (grant number 20120131110025). http://jme.endocrinology-journals.org DOI: 10.1530/JME-14-0019
() 2014 Society for Endocrinology Printed in Great Britain
Published by Bioscientifica Ltd 


\section{Author contribution statement}

W-L Z performed the major experiments, $C-Y L$ cloned the gene, $W L$ helped on EMSA, D W performed overexpression experiments, J-X W directed the study, and X-F Z designed the study and wrote the final version of the manuscript.

\section{Acknowledgements}

The nucleotide sequence of Met1 has been submitted to GenBank with GenBank Number: KJ184572.

\section{References}

Abdou MA, He Q, Wen D, Zyaan O, Wang J, Xu J, Baumann AA, Joseph J, Wilson TG \& Li S 2011 Drosophila Met and Gce are partially redundant in transducing juvenile hormone action. Insect Biochemistry and Molecular Biology 41 938-945. (doi:10.1016/j.ibmb.2011.09.003)

Ashok M, Turner C \& Wilson TG 1998 Insect juvenile hormone resistance gene homology with the bHLH-PAS family of transcriptional regulators. PNAS 95 2761-2766. (doi:10.1073/pnas.95.6.2761)

Beischlag TV, Wang S, Rose DW, Torchia J, Reisz-Porszasz S, Muhammad K, Nelson WE, Probst MR, Rosenfeld MG \& Hankinson O 2002 Recruitment of the NCoA/SRC-1/p160 family of transcriptional coactivators by the aryl hydrocarbon receptor/aryl hydrocarbon receptor nuclear translocator complex. Molecular and Cellular Biology 22 4319-4333. (doi:10.1128/MCB.22.12.4319-4333.2002)

Bitra K \& Palli SR 2009 Interaction of proteins involved in ecdysone and juvenile hormone signal transduction. Archives of Insect Biochemistry and Physiology 70 90-105. (doi:10.1002/arch.20281)

Cakouros D, Daish T, Martin D, Baehrecke EH \& Kumar S 2002 Ecdysoneinduced expression of the caspase DRONC during hormone-dependent programmed cell death in Drosophila is regulated by broad-complex. Journal of Cell Biology 157 985-996. (doi:10.1083/jcb.200201034)

Charles J-P, Iwema T, Epa VC, Takaki K, Rynes J \& Jindra M 2011 Ligand-binding properties of a juvenile hormone receptor, Methoprene-tolerant. PNAS 108 21128-21133. (doi:10.1073/ pnas.1116123109)

De Loof A, De Haes W, Janssen T \& Schoofs L 2014 The essence of insect metamorphosis and aging: electrical rewiring of cells driven by the principles of juvenile hormone-dependent $\mathrm{Ca}(2+)$-homeostasis. General and Comparative Endocrinology 199 70-85. (doi:10.1016/j.ygcen. 2014.01.009)

Dong D-J, Liu W, Cai M-J, Wang J-X \& Zhao X-F 2013 Steroid hormone 20-hydroxyecdysone regulation of the very-high-density lipoprotein (VHDL) receptor phosphorylation for VHDL uptake. Insect Biochemistry and Molecular Biology 43 328-335. (doi:10.1016/j.ibmb.2013.02.001)

Godlewski J, Wang S \& Wilson TG 2006 Interaction of bHLH-PAS proteins involved in juvenile hormone reception in Drosophila. Biochemical and Biophysical Research Communications 342 1305-1311. (doi:10.1016/ j.bbrc.2006.02.097)

Guo E, He Q, Liu S, Tian L, Sheng Z, Peng Q, Guan J, Shi M, Li K \& Gilbert LI 2012 MET is required for the maximal action of 20-hydroxyecdysone during Bombyx metamorphosis. PLoS ONE 7 e53256. (doi:10.1371/ journal.pone.0053256)

Hou L, Cai M-J, Liu W, Song Q \& Zhao X-F 2012 Small GTPase Rab4b participates in the gene transcription of 20-hydroxyecdysone and insulin pathways to regulate glycogen level and metamorphosis. Developmental Biology 371 13-22. (doi:10.1016/j.ydbio.2012.06.015)

Jindra M, Malone F, Hiruma K \& Riddiford LM 1996 Developmental profiles and ecdysteroid regulation of the mRNAs for two ecdysone receptor isoforms in the epidermis and wings of the tobacco hornworm, Manduca sexta. Developmental Biology 180 258-272. (doi:10.1006/ dbio.1996.0299)
Jindra M, Palli SR \& Riddiford LM 2013 The juvenile hormone signaling pathway in insect development. Annual Review of Entomology $\mathbf{5 8}$ 181-204. (doi:10.1146/annurev-ento-120811-153700)

Jones G \& Sharp PA 1997 Ultraspiracle: an invertebrate nuclear receptor for juvenile hormones. PNAS 94 13499-13503. (doi:10.1073/pnas.94. 25.13499)

Kayukawa T, Minakuchi C, Namiki T, Togawa T, Yoshiyama M, Kamimura M, Mita K, Imanishi S, Kiuchi M, Ishikawa Y et al. 2012 Transcriptional regulation of juvenile hormone-mediated induction of Kruppel homolog 1, a repressor of insect metamorphosis. PNAS 109 11729-11734. (doi:10.1073/pnas.1204951109)

Konopova B \& Jindra M 2007 Juvenile hormone resistance gene Methoprene-tolerant controls entry into metamorphosis in the beetle Tribolium castaneum. PNAS 104 10488-10493. (doi:10.1073/pnas. 0703719104)

Lam G, Hall B, Bender M \& Thummel C 1999 DHR3 is required for the prepupal-pupal transition and differentiation of adult structures during Drosophila metamorphosis. Developmental Biology 212 204-216. (doi:10.1006/dbio.1999.9343)

Lan Q, Hiruma K, Hu X, Jindra M \& Riddiford LM 1999 Activation of a delayed-early gene encoding MHR3 by the ecdysone receptor heterodimer EcR-B1-USP-1 but not by EcR-B1-USP-2. Molecular and Cellular Biology 19 4897-4906.

Li M, Mead EA \& Zhu J 2011 Heterodimer of two bHLH-PAS proteins mediates juvenile hormone-induced gene expression. PNAS 108 638-643. (doi:10.1073/pnas.1013914108)

Liu Y, Sheng Z, Liu H, Wen D, He Q, Wang S, Shao W, Jiang R-J, An S \& Sun Y 2009 Juvenile hormone counteracts the bHLH-PAS transcription factors MET and GCE to prevent caspase-dependent programmed cell death in Drosophila. Development 136 2015-2025. (doi:10.1242/ dev.033712)

Liu W, Zhang FX, Cai MJ, Zhao WL, Li XR, Wang JX \& Zhao XF 2013 The hormone-dependent function of Hsp90 in the crosstalk between 20-hydroxyecdysone and juvenile hormone signaling pathways in insects is determined by differential phosphorylation and protein interactions. Biochimica et Biophysica Acta 1830 5184-5192. (doi:10.1016/j.bbagen.2013.06.037)

Liu W, Cai M-J, Wang J-X \& Zhao X-F 2014 In a non-genomic action, steroid hormone 20-hydroxyecdysone induces phosphorylation of cyclin-dependent kinase 10 to promote gene transcription. Endocrinology 155 1738-1750. (doi:10.1210/en.2013-2020)

Minakuchi C, Xiaofeng Z \& Riddiford LM 2008 Krüppel homolog 1 (Kr-h1) mediates juvenile hormone action during metamorphosis of Drosophila melanogaster. Mechanisms of Development 125 91-105. (doi:10.1016/ j.mod.2007.10.002)

Minakuchi C, Namiki T \& Shinoda T 2009 Krüppel homolog 1, an early juvenile hormone-response gene downstream of Methoprene-tolerant, mediates its anti-metamorphic action in the red flour beetle Tribolium castaneum. Developmental Biology 325 341-350. (doi:10.1016/j.ydbio. 2008.10.016)

Murre C, McCaw PS \& Baltimore D 1989 A new DNA binding and dimerization motif in immunoglobulin enhancer binding, daughterless, MyoD, and myc proteins. Cell 56 777-783. (doi:10.1016/ 0092-8674(89)90682-X)

Riddiford LM, Hiruma K, Zhou X \& Nelson CA 2003 Insights into the molecular basis of the hormonal control of molting and metamorphosis from Manduca sexta and Drosophila melanogaster. Insect Biochemistry and Molecular Biology 33 1327-1338. (doi:10.1016/ j.ibmb.2003.06.001)

Riddihough G \& Pelham HR 1987 An ecdysone response element in the Drosophila hsp27 promoter. EMBO Journal 6 3729-3734.

Sevala V \& Davey K 1993 Juvenile hormone dependent phosphorylation of a $100 \mathrm{kDa}$ polypeptide is mediated by protein kinase $\mathrm{C}$ in the follicle cells of Rhodnius prolixus. Invertebrate Reproduction \& Development 23 189-193. (doi:10.1080/07924259.1993.9672314) 
Shao H-L, Zheng W-W, Liu P-C, Wang Q, Wang J-X \& Zhao X-F 2008 Establishment of a new cell line from lepidopteran epidermis and hormonal regulation on the genes. PLOS ONE 3 e3127. (doi:10.1371/ journal.pone.0003127)

Shin SW, Zou Z, Saha TT \& Raikhel AS 2012 bHLH-PAS heterodimer of methoprene-tolerant and Cycle mediates circadian expression of juvenile hormone-induced mosquito genes. PNAS 109 16576-16581. (doi:10.1073/pnas.1214209109)

Sun X \& Song Q 2006 PKC-mediated USP phosphorylation is required for 20E-induced gene expression in the salivary glands of Drosophila melanogaster. Archives of Insect Biochemistry and Physiology 62 116-127. (doi:10.1002/arch.20130)

Taylor BL \& Zhulin IB 1999 PAS domains: internal sensors of oxygen, redox potential, and light. Microbiology and Molecular Biology Reviews 63 479-506.

Wang S, Wang J, Sun Y, Song Q \& Li S 2012 PKC-mediated USP phosphorylation at Ser35 modulates 20-hydroxyecdysone signaling in Drosophila. Journal of Proteome Research 11 6187-6196. (doi:10.1021/ pr3008804)
Yao TP, Segraves WA, Oro AE, McKeown M \& Evans RM 1992 Drosophila ultraspiracle modulates ecdysone receptor function via heterodimer formation. Cell 71 63-72. (doi:10.1016/0092-8674(92)90266-F)

Zhang Z, Xu J, Sheng Z, Sui Y \& Palli SR 2011 Steroid receptor co-activator is required for juvenile hormone signal transduction through a bHLH-PAS transcription factor, methoprene tolerant. Journal of Biological Chemistry 286 8437-8447. (doi:10.1074/ jbc.M110.191684)

Zhao X-F, Wang J-X \& Wang Y-C 1998 Purification and characterization of a cysteine proteinase from eggs of the cotton boll worm, Helicoverpa armigera. Insect Biochemistry and Molecular Biology 28 259-264. (doi:10.1016/S0965-1748(98)00015-0)

Zhou B \& Riddiford LM 2001 Hormonal regulation and patterning of the broad-complex in the epidermis and wing discs of the tobacco hornworm, Manduca sexta. Developmental Biology 231 125-137. (doi:10.1006/dbio.2000.0143)

Zhu J, Chen L \& Raikhel AS 2003 Posttranscriptional control of the competence factor $\beta$ FTZ-F1 by juvenile hormone in the mosquito Aedes aegypti. PNAS 100 13338-13343. (doi:10.1073/pnas.2234416100)

Received in final form 16 May 2014

Accepted 27 May 2014

Accepted Preprint published online 27 May 2014
C 2014 Society for Endocrinology Printed in Great Britain
Published by Bioscientifica Ltd. 